ISSN: $1130-3743$

\title{
EL APRENDIZAJE ÉTICO Y LA FORMACIÓN UNIVERSITARIA: MÁS ALLÁ DE LA CASUALIDAD
}

\author{
Ethical development and university education: more \\ than a coincidence
}

\section{L'apprentissage éthique et la formation universitaire: au-delà du basard}

\section{Francisco ESTEBAN* y M. Rosa BUXARRAIS**}

* Universitat Internacional de Catalunya. Facultat d'Humanitas. C/ Inmaculada, 22.08017 Barcelona. Correo-e: festeban@cir.unica.edu

** Universidad de Barcelona. Facultat de Pedagogía. Departament de Teoria Història de l'Educació. Passeig de la Vall d'Hebró, 171. 08035 Barcelona.

Fecha de recepción: marzo de 2004

Fecha de aceptación definitiva: octubre de 2004

BIBLID [(1130-3743) 16, 2004, 91-108]

\section{RESUMEN}

En el presente trabajo tratamos de abrir nuevos caminos de reflexión a uno de los temas menos atendidos en el discurso contemporáneo sobre la Educación Superior: el desarrollo ético de los estudiantes universitarios. Y es que, sorprendentemente, la formación ética de nuestros estudiantes parece haber sido olvidada y desatendida pedagógicamente hablando. Tanto es así, que tenemos la sensación de que la relación entre enseñanza universitaria y aprendizaje ético se caracteriza por la casualidad, por el mero azar. Dicho esto, y a sabiendas de la necesidad que conlleva el siglo xxI de que dicha relación sea algo más que una casualidad, tratamos de aclarar lo que entendemos por aprendizaje ético así como las condiciones del contexto universitario que podrían facilitar dicho aprendizaje.

Palabras clave: aprendizaje ético, formación universitaria, ciudadanía. 


\section{SUMMARY}

This article discusses one of the most disregarded topics in the contemporary debate about Higher Education: the ethic development of students. Surprisingly enough, from an educational perspective, this issue seems to have been ignored. So much so, that the sensation is that the relationship between university education and ethical development is considered a mere coincidence. Given the importance of this issue in the $21^{\text {st }}$ Century, the aim of this article is to clarify the concept of ethical development, as well as the factors and circumstances that facilitate its growth.

Key words: ethical development, university education, citizenship.

\section{SOMMAIRE}

Avec ce travail, nous essayons d'ouvrir de nouvelles voies de réflexion sur l'un des thèmes les moins étudiés du discours contemporain sur l'Éducation Supérieure: le développement éthique des étudiants universitaires. En effet, il est surprenant que la formation éthique et morale de nos étudiants semble avoir été oubliée et non traitée pédagogiquement parlant. Et ce, à tel point, que nous avons la sensation que la relation entre enseignement universitaire et apprentissage éthique se caractérise par le hasard, par le pur hasard. Ceci dit, connaissant la nécessité, qu'entraîne le xxI ${ }^{e}$ siècle, que cette relation soit plus qu'un hasard, nous essayons d'éclaircir ce que l'on entend par apprentissage éthique et aussi par les conditions du contexte universitaire que pourraient faciliter le dit apprentissage.

Mots clef: apprentissage éthique, formation universitaire, citoyenneté.

Por todos los que nos dedicamos al mundo de la formación universitaria y además nos interesamos por ella, son conocidos los avatares de la relación entre la enseñanza y el aprendizaje. La mayoría de las veces y, parafraseando al filósofo, sólo sabemos que no sabemos nada. Y es que un propósito de enseñanza universitaria no tiene por qué llevarnos sí o sí a la meta del aprendizaje pretendido. Basta con desviarse, aunque sea ligeramente, del camino de la enseñanza magistral y del corpus curricular establecido para caer en la cuenta de que se ha entrado en un laberinto inhóspito y sin salida a la vista.

Efectivamente, estamos rodeados de intentos de nuevas andaduras en la docencia universitaria que han caído en tierras movedizas y en los que no ha germinado la semilla de los aprendizajes en un principio deseados. Algunas veces incluso han dado frutos por nadie esperados. Por el contrario, otros tantos intentos deberían ser aplaudidos tanto por sus frutos recogidos como por la sensatez del proceso de enseñanza, aprendizaje y evaluación llevado a cabo. Innovar, incluso en la enseñanza universitaria, es escoger nuevos caminos previo ejercicio de reflexión y planificación, condición sine qua non se va abocado al laberinto. No es objeto de este trabajo analizar las causas que enturbian la relación entre la 
enseñanza y el aprendizaje en las cruzadas innovadoras de la universidad de hoy, aunque apostamos que el propio ethos universitario es la causa principal (Hoyos, 2004). Fuera lo que fuere, no es un problema nuevo y, a buen seguro, tampoco estarían ausentes de situaciones problemáticas las lectio y las disputatio que daban sentido a la pedagogía escolástica de las primeras universidades, y aún así, a trancas y barrancas, hemos ido avanzando.

Una vez más es el maestro Ortega quien nos muestra con facilidad la complejidad. En la universidad se debería enseñar lo que realmente se debe aprender, ni más ni menos (Ortega y Gasset, 1930). Por derivación gramatical, nacen dos preguntas a partir de la máxima planteada por el filósofo y que serán tratadas respectivamente en los dos primeros apartados del presente trabajo.

La primera de las preguntas: ¿Qué se debe aprender en la universidad de hoy? Parecen claras y oportunas las indicaciones de la UNESCO según las que el aprendizaje del siglo xxI ha de ser un aprendizaje para toda la vida (UNESCO, 1997). Efectivamente, el caminar por la universidad debería ser un proceso de aprendizaje significativo y lleno de sentido. Conceptos, hechos, paradigmas, teorías, estrategias de aprendizaje autónomo, competencias profesionales..., en definitiva todo aquello que sea útil para toda una vida personal y profesional, cosa que ocurre cuando se aprende a aprender y también a desaprender. Nos referimos a un enfoque profundo del aprendizaje que aspire a algo más que el rendir cuentas el día del examen. Y aunque lo dicho parece indiscutible, no es suficiente. Nunca fue válido un médico, un arquitecto o un maestro con muy buenas aptitudes profesionales pero con ceguera ética, tanto en el desarrollo de la profesión como delante de los problemas de la comunidad. No digamos hoy en día, en tiempos de incertidumbre, donde hay más interrogantes que certezas, donde el pensamiento newtoniano propio del siglo xx parece que se aleja y nos va a tocar pensar a tientas (Galbraith, 1982; Escotet, 2004). El conocimiento propio de la educación universitaria necesita ser utilizado bajo principios éticos que ayuden a consolidar la moralidad del estudiante.

El aprendizaje para toda la vida, propio de la universidad, debería ser además el aprendizaje de uno mismo, la construcción de una personalidad moral (Puig, 1996) que fue gestada en niveles inferiores del sistema educativo pero que debería madurar en la formación universitaria. El aprendizaje universitario no debería ser la formación de un médico, arquitecto o maestro cualquiera, sino la formación de un médico, arquitecto o maestro con altura moral. El uso de la medicina, la arquitectura o el magisterio sin moralidad, puede ser desastroso para la comunidad y penoso para aquellos que de tal modo ejerzan su profesión. Ejemplos tenemos todos los que queramos. Así pues, el aprendizaje que nos permite ejercer una profesión debería ir acompañado del aprendizaje ético que nos permite ejercer la ciudadanía. Se trata de considerar ambos tipos de aprendizaje como dos caras de una misma moneda (Esteban, 2004).

Nos planteamos ahora la segunda de las dos preguntas anunciadas: ¿Qué se debería enseñar en la universidad? Por lógica pedagógica, se debería enseñar todo 
lo dicho anteriormente, ni más ni menos. El profesional del siglo que ya está aquí, nos lo imaginamos bien enseñado, es decir, que ha recibido las ayudas psicopedagógicas necesarias para tener la mente bien ordenada (Morin, 2000), para acumular muchas y variadas cosas y para aprender y desaprender de manera autónoma cuando tenga razones para ello. Por definición, una nueva cultura del aprendizaje exige una nueva cultura de la enseñanza.

En nuestro terreno, el de la ética y la moral, no cometamos el catedralicio error de pensar que una personalidad moral se instalará en las mentes y corazones de nuestros estudiantes universitarios por añadidura de otros aprendizajes de carácter científico y humanístico. Incluso la transmisión de códigos éticos y deontológicos de las profesiones aun siendo un avance psicopedagógico plausible no debería contentarnos. La enseñanza de la ética debería acicalar y dar color a toda la vida universitaria y no ser tratada como un conjunto de créditos, usualmente en forma de asignatura optativa, en los últimos años de carrera. En definitiva, si un comportamiento ético debe ser aprendido, debería, por ende, ser enseñado y teniendo en cuenta dicha máxima deberíamos organizar el escenario educativo universitario (Martínez, Buxarrais y Esteban, 2002).

El tema planteado, a saber, la formación ética y moral en la universidad, se nos representa lo suficientemente importante como para dejarlo en manos de la casualidad o del buen hacer de unos pocos sensibilizados con la causa. Debería tal objetivo dar sentido a todo un proceso de atención psicopedagógica en un contexto universitario.

El tercer apartado del presente trabajo es, precisamente, un intento de vislumbrar los pasos que podrían llevarnos a que el aprendizaje ético y la formación universitaria se encuentren en el terreno de las intenciones psicopedagógicas de los planes de estudios de Educación Superior. Es así como evitaremos que se encuentren, en el mejor de los casos, en el terreno de la casualidad.

En este sentido proponemos que la comunidad académica piense en el modelo de ciudadano universitario que pretende ofrecer a la comunidad social y profesional. Lo conocido como extensión de la cultura universitaria exige pensar en el médico, arquitecto y maestro que llevará la cultura y el conocimiento, adquiridos en la universidad, a todos los rincones de su comunidad con tal de aportar soluciones razonadas y éticas a los problemas planteados. También proponemos que la comunidad académica planifique el proceso de enseñanza, y por ende el de aprendizaje, de una vida universitaria con sentido ético que garantice una formación moral de los estudiantes. No en vano, también la construcción de la personalidad moral, como cualquier otra área de conocimiento, necesita un proceso formativo secuenciado y contextualizado. No nos debería saciar la implementación de una asignatura optativa en los últimos coletazos de vida universitaria, por muy bien que ésta sea planteada y llevada a cabo. Un aprendizaje que cala fondo necesita un proceso que fomente tal calado. Por último, proponemos que la comunidad universitaria actúe mediante una pedagogía propia del aprendizaje ético. Las intenciones pensadas, pero que no proponen una praxis 
concreta, se desvanecen y pronto se olvidan. La vida universitaria debería estar plagada de prácticas educativas pensadas también en términos de formación ética. Así pues, pensar, planificar y actuar son tres acciones que pueden ayudar para que la relación entre el aprendizaje ético y la formación universitaria sea algo más que una casualidad en el caso de que ésta se produzca.

\section{El APRENDIZAJE ÉTICO EN LA UNIVERSIDAD DE HOY}

Nos referimos en este apartado a la primera de las cuestiones planteadas en los inicios del presente trabajo, es decir, la consideración del aprendizaje ético en la universidad de hoy. Como hemos tratado de señalar, ya no basta únicamente con disponer de una acumulación de conocimientos científicos y humanísticos al más alto nivel, por muy amueblados que éstos se encuentren en las mentes de los estudiantes. El caminar por la universidad debería también ser una experiencia de vida, de aprendizaje ético y desarrollo de la personalidad moral. Efectivamente, participar en una universitas, a saber, en una corporación de maestros y estudiantes que comparten un proyecto común y una identidad particular, debería conducir a un cambio, a una transformación de los participantes. Recordemos que transformar en lenguaje educativo es mucho más que informar y deformar (Torralba, 2002). En toda transformación se produce un cambio. Alguien transformado no es el mismo que fue. Sirva de ejemplo: Alonso Quijano no es el mismo al inicio que al final del Quijote, se ha transformado en otra persona, principalmente por su relación con el bueno de Sancho. Y éste también ha sido transformado debido al contacto permanente con su señor y protector.

En lenguaje psicopedagógico deberíamos hablar de transformación del estudiante porque la vida universitaria puede aportar aquellas experiencias de aprendizaje que vayan rellenando el libro de la narración personal, el texto de vida de cada uno de los aprendices universitarios (Sennet, 2003). Nos referimos a experiencias que sean vividas y sentidas con carácter de recuerdo, y no tanto de reminiscencias. En este último caso, el acceso a la universidad, y por lo tanto al conocimiento, queda relacionado con una cuestión de actividad productiva, un control o mecanismo de ascenso social y deja de tener sentido para el desarrollo ético y moral de las personas (Habermas, 1984, 1987). Es entonces cuando el aprendizaje universitario deja de transformar a los estudiantes y deviene una circunstancia, una colección de anécdotas fáciles de olvidar y que difícilmente transforman a quien las vive o, según como se mire, las sufre. Así pues, defendemos que el contexto universitario que acompaña las narraciones de vida de los estudiantes sea formativo, que tenga que ver con el aprendizaje de la persona, con la construcción de la personalidad moral.

Pero, ¿qué aprendizaje ético tiene sentido en la universidad de hoy?, ¿qué semántica deberían tener las diferentes experiencias universitarias por las que pasa el estudiante para que éste sea transformado en clave ética? Creemos que tiene 
sentido pensar en algo más que lo que se conoce como crédito y para nosotros es una reducción del concepto aprendizaje al servicio del sistema productivo. Tampoco somos partidarios de la ascensión al mundo de las ideas en lo que al aprendizaje ético en la universidad se refiere, precisamente su platonismo y romanticismo poco pragmático acaba convirtiéndose en narcisismo de la universidad. Como casi siempre ocurre, deberíamos situarnos en el punto medio. Ya lo hemos dicho, sin intenciones educativas pensadas desde el ejercicio de la reflexión no hay pedagogía universitaria y sin una didáctica de la dimensión moral de los estudiantes no hay concreción de las intenciones antes tomadas. La no relación de ambos extremos deviene, o bien una realidad que se caracteriza por un discurso muy atractivo para los oídos pero con una nulidad pedagógica espectacular, o bien una cruzada personal de unos pocos profesores identificados con la causa pero condenada a la desaparición. Deberíamos lanzar el ancla en el punto medio, entre el reduccionismo didáctico nihilista y el discurso demasiado alejado de la realidad universitaria. Punto medio que no es otra cosa que las relaciones que se establecen entre profesores y estudiantes.

Proponemos cuatro corolarios de aprendizaje ético con la mirada puesta en el ciudadano universitario que soñamos y deseamos para el siglo que ya está aquí. Así pues, el aprendizaje ético y moral en la universidad de hoy debería ser el aprendizaje de la acogida, el de la responsabilidad, el de la profesión de la profesión y el de saberse universitario.

\subsection{El aprendizaje de la acogida}

El aprendizaje de la acogida es pensar una manera diferente de relación que la institución entabla con sus estudiantes. Reducir y vulgarizar toda relación universitaria, como de hecho ocurre, a una cuestión administrativa, nuevamente al servicio del sistema productivo, nos hace desperdiciar la oportunidad de disfrutar del placer de sentirse acogido en un contexto universitario. Defendemos una relación de acogida que supere el frío formalismo y el academicismo más absurdo. El aprendizaje universitario, que es el aprendizaje del conocimiento y la cultura al más alto nivel, debería desarrollarse en el terreno de la acogida, de la fe en el otro. En definitiva, el acto educativo es un acto de fe en todos los niveles (Torralba, 2001). No olvidemos que la idea universidad nació de una relación de acogida entre docentes y discentes en torno a la verdad y el conocimiento (Iyanza, 2000). Una relación de fe, comprometida y responsable, en la búsqueda de la verdad y el conocimiento. Así quedó reflejado en la Universidad de Bolonia en el siglo XIV cuando los estatutos del Collegium Doctorum concretaron la relación de acogida entre profesores y estudiantes con un compromiso explícito sobre la preparación de las lectio, sobre el estar disponibles en todo momento, sobre el llegar puntualmente a clase, etc. Una acogida comprometida y con fe hacia el otro y su acción. 
Quien ha sido acogido desde el conocimiento y la cultura está en disposición de acoger. Pensamos que la narración de vida de los estudiantes universitarios debería adquirir un papel de gratuidad para que el otro disponga de dicha narración que, no olvidemos, forma parte de un proyecto común. En época de incertidumbre, más que nunca, todos somos uno. La vida universitaria no es una narración con un final anunciado, es decir, que acaba tras el último año de carrera. El aprendizaje para toda la vida debería estar abierto a nuevas relaciones y experiencias de enriquecimiento personal, a nuevas oportunidades de acogida gracias a lo aprendido más allá del campus. La bondad de esta apertura narrativa, de esta co-construcción inacabada no es posible sin el sentido de gratuidad. La apuesta de las ahora en construcción comunidades universitarias ${ }^{1}$ por la formación de ciudadanos implicados, comprometidos y solidarios pasa por esta relación de gratuidad. Si somos acogidos acogeremos también en la universidad y también desde el conocimiento. La semántica de las narraciones de vida universitarias abiertas y libres, pueden incorporar la gratuidad y llegar a ser narraciones abiertas y desinteresadas para el otro que la necesite. En otras palabras, pueden llegar a ser proyectos de vida ciudadana en actitud de interés por el otro.

La acogida universitaria que será experiencia de vida para el estudiante, también debería ser respetuosa. Una acogida disfrazada y sin respeto por el aprendizaje del discente es adoctrinamiento, nos guste o no. Un respeto unidireccional tampoco es respeto, es una relación de abuso. Muy fácil lo tenemos los docentes universitarios para abusar desde la tarima y el conocimiento. Sólo quien ha gozado del aprendizaje de la acogida en su sentido más profundo, podrá después atender la mirada del otro (Levinas, 1993) como ciudadano universitario sin adoctrinar ni abusar.

\subsection{El aprendizaje de la responsabilidad}

El filósofo francés Paul Ricoeur nos sugiere a través de su obra que deberiamos actuar siempre como si un testigo imaginario estuviera escuchando lo que decimos $\mathrm{y}$ viendo lo que hacemos. También lo que nos callamos y de lo que nos escaqueamos. Añade el pensador que deberíamos pensar en un testigo que además de escuchar y ver está a la espera de nuestros pensamientos y acciones (Ricoeur, 1900). Se puede decir más alto pero no más claro. Se nos ocurre que el aprendizaje universitario también debería estar fundamentado en este testigo imaginario ahora que el viento del individualismo liberal empieza a soplar fuerte. El estudiante universitario debería preguntarse ¿Y a mi, en la comunidad, quién me necesita? (Sennet, 2000, 154).

1. No es difícil extraer esta lectura de las últimas declaraciones del Espacio Europeo de Educación Superior, especialmente del documento de Bolonia (1999) y los que han ido apareciendo uno detrás de otro, Lisboa (2000), Praga y Salamanca (2001), Graz y Berlín (2003). 
No deberían ser válidos proyectos de vida universitaria independientes a estas alturas de la historia, en un mundo interdependiente como el actual. Los ciudadanos universitarios que miran la cultura y el conocimiento cara a cara, de arriba abajo, que lo degustan, lo visten y desvisten desde diversas disciplinas académicas, tienen la posibilidad de abrir las puertas de la cultura y la razón a los "testigos" que socialmente las tienen cerradas. Se trata de atender la mirada del otro (Levinas, 1993) desde la formación universitaria. Creemos que la formación universitaria pasa ineludiblemente por esta ética de la responsabilidad hacia la comunidad (Jonas, 1995). El aprendizaje universitario debería de tener que ver con la inteligencia sentiente (Zubiri, 1986) y dejar sabor de responsabilidad comunitaria y de compromiso para toda la vida. Por esta razón, resulta interesante para el tema que nos ocupa la segunda parte de la aportación de Ricoeur, cuando advierte que el testigo imaginario confía en nosotros y nuestras acciones. Así es cómo éste se vuelve real, con nombres y apellidos.

En definitiva, el ciudadano universitario que camina por la universidad debería redactar su narración de vida teniendo siempre presente el testigo que es real, y por desgracia, numeroso. Son muchas las comunidades y personas que necesitan para sus problemas, soluciones razonadas y razonables, es decir, que provengan de mentes y corazones universitarios. En este sentido, y con razón, se muestra especialmente contundente el propio Habermas cuando sugiere que quien conscientemente excluye al otro, que tiene derecho a estar, se está traicionando a sí mismo (Habermas, 2000). Doblemente se traiciona, diríamos nosotros, si lo hace desde el poder y prestigio que otorga la formación universitaria. Deberían alertarnos estudios según los cuales, la juventud, incluida la universitaria, no relaciona su proyecto de vida con la interrelación con los demás. El concepto de felicidad de la juventud actual, según la última encuesta europea de valores, no incluye la responsabilidad con el otro (VV.AA., 2002).

El aprendizaje de la responsabilidad con la comunidad es algo más que el mero voluntarismo, por otra parte necesario. Se debería incorporar dicha intención formativa en el corpus curricular académico y en la cultura universitaria. El ciudadano universitario tomará conciencia de su papel en la construcción de una sociedad más justa y equitativa cuando se dé cuenta de que desde la cultura y el conocimiento debería actuar sobre la comunidad porque hay alguien que lo necesita (Quintanilla, 1999). ¿No es ésta una razón de peso para pensar en el aprendizaje ético en la universidad?

Así pues, el aprendizaje universitario debería incorporar la dimensión de la responsabilidad comunitaria. De otra manera, nos cargamos de razones para volver a hablar de los scholari simplex, aquellos estudiantes medievales para los que el paso por la universidad era un pretexto para la buena vida y así poder vivir una aventura con fines absolutamente individualistas. El aprendizaje universitario del siglo XXI debería ser el aprendizaje de la sensación de problemas (Ortega y Gasset, 1930). El aprendizaje que inquieta a la persona a querer cambiar el mundo desde la profesión, la cultura y el conocimiento. Creemos que la responsabilidad del 
ciudadano universitario acaba donde esté el otro que necesita ayuda proveniente de una mente y un corazón universitarios.

\subsection{El aprendizaje del sentido de la profesión}

En el siglo de la técnica, de los técnicos y de los tecnicismos, el aprendizaje de la profesión corre el peligro de no llegar a la profundidad que consideramos necesaria en la universidad de hoy. El estudiante universitario puede convertirse fácilmente, y casi sin darse cuenta, en un eslabón más de una cadena donde puede desarrollar su profesión pero que ha perdido el sentido de su quehacer. Dicho de otra manera, se puede convertir en un terminal más del sistema productivo (Castells, 1997). El universitario, ya profesional, puede ser como aquella hormiga que va en medio de la hilera, que no sabe dónde se dirige ni recuerda de dónde ha salido. Deberíamos ir con cuidado con la corriente de viento nihilista que sopla en la actualidad en el hecho universitario.

La semántica del propio concepto profesar nos puede ser útil. Professare es declarar abiertamente la fe, sentir intensamente un amor, una inclinación. Profesión es la acción de profesar. La profesión pues va más allá de la técnica, de un conjunto de habilidades profesionales. Demasiado entusiasmados estamos con las famosas competencias en la universidad de hoy, y corremos el peligro de ver únicamente la punta del iceberg y no toda la sustancia que la sustenta. ¿Volveremos una vez más a seleccionar las habilidades para cada profesión y así poder dibujar a los nuevos técnicos cada vez más especializados? Podemos pensar que sí, pero con esto no será suficiente. El aprendizaje de una profesión debería superar la adquisición de un puñado de competencias. Consideramos la profesión como un acto de fe del trabajo, del oficio, en definitiva, la profesión de la propia profesión (Derrida, 2002).

El aprendizaje universitario, en sentido ético y moral, debería incluir la degustación de la esencia del concepto profesión de todas y cada una de sus especialidades. Los profesionales universitarios no deberían ser eslabones de la cadena que mejores condiciones ofrezca, de la misma manera que su actuación y pensamiento no debería agotarse en la técnica. Al mismo tiempo las organizaciones empresariales tampoco deberían confiar únicamente en técnicos por muy competentes que éstos sean. La profesión de la profesión es ponerse codo con codo con la verdad, la cultura y el conocimiento. Exige salir del imago mundi siempre maniqueo y ver la realidad en toda su complejidad. Profesar la profesión es tomar posición, desde el conocimiento que da sentido a un ámbito profesional y desde la cultura que ilumina todas las realidades posibles. La profesión de la profesión no es preguntarse si se quiere ser médico, arquitecto o maestro sino qué tipo de médico, arquitecto o maestro se quiere llegar a ser. Creemos que la formulación de esta pregunta brilla por su ausencia en la paideia universitaria de nuestros días. Desde el conocimiento científico y humanístico propio de la 
universidad hemos fabricado los instrumentos más mortíferos y hemos elaborado los discursos políticos más aberrantes. Estas acciones y pensamientos que han provocado la barbarie y han contado con el apoyo de instituciones universitarias también deberían ser explicadas en nuestros días. También se aprende cuando se muestran los caminos por los que no se debería volver a transitar. En otras palabras, el aprendizaje de una profesión es el aprendizaje de su sentido de ser y hacer, del tratamiento de los dilemas éticos y morales que en ella se puedan dar.

Así pues, el aprendizaje de la profesión de la profesión es el aprendizaje de la soberanía personal que en su día reclamó Francisco Giner de los Ríos. El médico, el arquitecto y el maestro pueden ser vistos como profesionales de diferentes ámbitos de conocimiento o como ciudadanos abanderados que dan sentido y razón de ser de una profesión, cada uno de la suya.

\subsection{El aprendizaje del saberse universitario}

Desde que la idea universidad germinara, allá por el siglo xI, el universitario ha sido considerado una persona con juicio para hacer y decir. En otras palabras, un ciudadano con estudios universitarios es considerado una fuente de buen juicio para su comunidad, un ejemplo de buenas maneras, de magistral razonamiento..., en definitiva, un bomo educatus. La universidad ha significado pues un mecanismo de transformación de sus usuarios. Quien ha caminado por la universidad es supuestamente alguien transformado en una persona con buena educación, con juicio de causa sobre la realidad y con opiniones aplaudidas por la comunidad. Decimos supuestamente porque algunas veces la realidad supera la ficción.

Los inicios de la idea universidad ya se encargaron de conseguir tal fin introduciendo en sus planes de estudio la gramática, la retórica y la dialéctica. Los estudiantes de entonces caminaban por instituciones educativas que trabajaban de manera explícita el arte de saberse universitario, es decir, el arte de hablar, discernir, opinar... También eran éstas las aptitudes y actitudes que el estudiante valoraba de sus profesores.

Fuere como fuere, son cada vez más numerosas y altaneras las voces que se alarman y escandalizan, no sin razón, por la devaluación de las buenas maneras, del saberse universitario, en nuestros días. Se habla incluso de la infantilización del estudiante (Torrents, 2002, 34). Seamos sinceros, el espectacular crecimiento cuantitativo de instituciones universitarias en nuestro territorio no ha significado un aumento de las buenas maneras entre la ciudadanía universitaria. Todo lo contrario. En este sentido, haría falta ver si ha sido peor el remedio que la enfermedad. Autores como Savater proponen de forma irónica, pero acertada, la denuncia en juzgados de guardia de estudiantes con formación universitaria que presenten niveles alarmantes de mala educación (Savater, 1996). Aun así, consideramos que la comunidad social continúa confiando en el bomo educatus que puedan ofrecerle las universitas allí instaladas. 
En cualquier caso, no pretendemos proponer un catálogo de criterios para el aprendizaje de las buenas maneras. Sería apostar por una pedagogía maniquea del tipo éste comportamiento es válido y éste otro debería ser fulminado. La universidad por definición da acogida a diferentes estudiantes, narradores y proyectos de vida, narraciones. Esto es lo que la convierte en la universidad abierta (Quintanilla, 1999). Pero tampoco pretendemos renunciar a los mínimos que precisamente la convierten en una institución social única e irrepetible. Pero, ¿cuáles son estos mínimos? Quizás en este punto se abre otra discusión que no forma parte de las intenciones del presente trabajo aunque la encontramos del todo necesaria y urgente. La deseada prudencia, el silencio oportuno, la argumentación razonada, el respeto hacia uno mismo y hacia los demás, saber dar las gracias, la buena disposición, la mirada atenta... son actitudes que deberían formar parte del saberse universitario. El pasotismo, la voz criticona que nada aporta, el ruido siempre molesto, el humo dentro del aula, los libros de todos embadurnados del fluorescente de algunos, el uso indiscriminado de las fotocopiadoras que amputan el conocimiento... deberían desaparecer del saberse universitario. A fin de cuentas, lo dicho, también se aprende.

\section{LA ENSEÑANZA ÉTICA Y MORAL EN LA UNIVERSIDAD DE HOY}

Si nos parecen importantes los aprendizajes éticos y morales que se deberían fomentar desde la universidad, no nos parecen menos el contexto y las condiciones donde dichas experiencias de aprendizaje y desarrollo personal tienen cabida. Tenemos la impresión de que el hecho universitario se ha volcado en la parcela del aprendizaje, especialmente durante la eclosión en las últimas décadas de la investigación psicoeducativa (Brockbank y McGuill, 2002). Investigación del todo necesaria pero incompleta y atrevida en conclusiones si no se considera el contexto ${ }^{2}$ en el que se producen los aprendizajes tan deseados.

Creemos que las maneras como la tramoya universitaria ${ }^{3}$ organiza el escenario formativo es clave para que se puedan representar un determinado tipo de escenas u otro, y que, por lo tanto, conduzcan al desenlace de la obra programado, o a otro por nadie esperado. En este sentido, vale la pena decir que la obra universitaria, desde sus inicios medievales, ha pretendido un desenlace próximo a la construcción de personas con conocimiento y cultura, personas críticas y en actitud de búsqueda de la verdad. En cambio, el escenario universitario contemporáneo, salvo excepciones difíciles de encontrar, ha sido organizado para actos de auténtica

2. Entendemos por contexto el conjunto de circunstancias que rodean y explican un acontecimiento, una situación, un aprendizaje.

3. Nos referimos con "tramoya universitaria" a administraciones educativas, responsables académicos, órganos de gobierno, docentes universitarios, personal de administración y servicios... todos los que forman parte del teatro educativo universitario. 
y exclusiva transmisión de conocimientos. Recordemos que la transmisión per se no inquieta la mente ni transforma la persona, simplemente la llena de conocimientos.

La historia de la universidad europea nos ha ofrecido tres grandes modelos de formación, tres maneras de organizar el escenario universitario (Bricall, 1997). El modelo universitario francés o napoleónico, controlado por el Estado, con gobierno académico jerárquico y burócrata que controla la formación de los futuros ciudadanos universitarios. Modelo que consideramos poco respetuoso con la autonomía universitaria en todos los sentidos y que cierra puertas a la búsqueda de la verdad. El modelo universitario inglés abandera la apuesta por la educación liberal. Es sin duda un instrumento seductor y paradisíaco para los defensores de un mundo ilustrado. El crecimiento intelectual y la búsqueda de la verdad son sus intenciones de formación. No obstante, consideramos que es una manera fácil de caer en el individualismo, absurdo para nuestros días, y de aumentar la miopía del compromiso ciudadano del estudiante universitario. El modelo universitario alemán ofrece sus servicios a la ciencia y su desarrollo, incluso por encima de la cultura a sabiendas del error que esto puede representar. El servilismo al mundo científico puede hacer perder el norte de la formación universitaria, de los aprendizajes éticos y morales mencionados en el apartado anterior.

Dicho esto, pensamos que la universidad de hoy debería considerar el proceso de formación sobre la base de unos criterios que nos permitan, precisamente, acercarnos al tipo de aprendizaje ético tratado en el apartado anterior. Así pues, consideramos que la enseñanza de la universidad de hoy debe darse en un contexto que atienda a los criterios de apertura, de extensión y de reflexión a tientas.

\subsection{La apertura de la universidad de boy}

Ya hemos señalado que la experiencia universitaria se nos representa como una experiencia de acogida desde el conocimiento y la cultura, y que hemos titulado en el apartado anterior como el aprendizaje de la acogida. Los ciudadanos universitarios deberían escribir sus narraciones personales con dicha experiencia vivida y sentida. Para alcanzar tal objetivo, el transcurso de las últimas décadas ha apostado por la apertura universitaria. Tanto es así que son cada vez más las sucursales de universidades extendidas a lo ancho y largo del territorio europeo. De todas formas ha sido, en el mejor de los casos, una apertura administrativa, una mejor manera de expedir títulos homologables. Aun así es una apertura que ha favorecido el intercambio de estudiantes y que se identifica con una de las demandas que llegan desde el horizonte Bolonia. También en los studium generale se otorgaba el licentia ubique docenci que tenía validez y reconocimiento en los más prestigiosos studium del territorio europeo medieval (Claramunt, 2002). Nuevamente defendemos la bondad de la idea pero no la consideramos suficiente.

La apertura de la universidad implica que las diferentes instituciones universitarias entren en un juego de complicidades, se reconozcan unas a otras en 
algo más que en la homologación de títulos, en un conjunto de asignaturas, en unos meses de estancia contabilizados en horas de enseñanza. La apertura que defendemos es algo más que reconocer, es compartir. La enseñanza universitaria debería participar de una misma misión y de unas mismas intenciones, lo que no implica, ni por asomo, perder la autonomía que ya ganamos hace unos años. La apertura universitaria de una comunidad es tratar de hacer algo conjuntamente, especialmente en la comunidad. En este sentido, la asunción de un modelo u otro de organización y de política universitaria tiene mucho que decir para que la apertura universitaria hacia la comunidad sea realidad o solamente sea un sueño. Tener un proyecto universitario a nivel de comunidad, ya no digamos de institución, nos parece un primer paso esencial para ello. Aplaudimos la idea de la Europa universitaria, siempre y cuando se implique a la comunidad en la formación de los ciudadanos universitarios, y es que la manera como se organiza el hecho universitario de una comunidad tiene mucho que ver con lo que realmente acaba pasando entre docentes y estudiantes en el aula.

Parece pues razonable que si dibujamos entre todos un esbozo de ciudadano universitario también podríamos ponernos manos a la obra en el diseño abierto y flexible de la paideia universitaria. A nuestro modo de ver, no son válidas las acciones puntuales, las asignaturas aisladas, o la muy buena voluntad de un grupo de profesores. Esto puede quedar muy lejos de la creación de una cultura de enseñanza compartida, de una apertura del campus universitario en el sentido más profundo del término (Pozo y Monereo, 2002). Tampoco deberíamos conformarnos con una institución universitaria que recela de sus méritos y no los hace públicos. El aislamiento y la cerrazón no es aconsejable en tiempos de interrelación. Apostamos por una apertura de las instituciones universitarias que mediante un dialogo genuinamente habermasiano (Habermas, 1954), es decir, sin la exclusión de nadie, hable sobre qué tipo de ciudadanos profesionales se pretenden, y consecuentemente, se prepare el escenario educativo para ello. Al mismo tiempo, apostamos porque la esencia de un proyecto compartido se pueda identificar de diferentes maneras en cada aula, departamento e institución universitaria. Autonomía coordinada e interrelacionada, compartir y no competir. Defendemos una enseñanza universitaria que permita escribir narraciones personales de vida insertas en un proyecto común, en nuestro caso en un proyecto de ciudadanía europea. Narraciones que sean escritas de una manera abierta, flexible y en las que todas las instituciones universitarias se vean representadas, las públicas y las privadas, las de carácter laico y las de opción confesional. Se trata de respetar todas las opciones que merecen ser respetadas.

\subsection{La extensión de la universidad de boy}

Se nos escapa a menudo que la comunidad universitaria es un número ingente de personas con presencia en todo el orbe. Se parecen los universitarios a los 
miembros de la secta del Fénix descrita por Borges (Borges, 1972). Así como no hay un grupo humano donde no figuren partidarios del Fénix, no hay comunidad de personas donde no figuren universitarios. Recordará el lector que el misterio de los miembros del Fénix es que comparten un mismo secreto y lo llevan allá donde llegan. ¿Actúa la universidad como la secta del Fénix? Cuantitativamente sí. Sería difícil no encontrar un espacio comunitario o sector profesional donde no halláramos una persona con experiencia universitaria. La contemporánea universidad de masas ha permitido el acceso a la vida universitaria a sectores de la población que no gozaron de ella en otros tiempos. En este sentido el universitario está presente en la comunidad, en cualquier rincón de ella.

Pero, ¿es suficiente con estar presente? Absolutamente no. Los miembros del Fénix no sólo estaban presentes, sino que eran reconocidos por ser miembros de un grupo, con sus maneras de ser y de hacer. Estar presente sea en la comunidad sea en el trabajo, es condición de ejercer lo aprendido en la universidad pero no es garantía de ello. El ciudadano universitario además de estar presente debería ser reconocido como tal, como alguien con altura ética y moral que llega cargado de posibles soluciones para la comunidad. En este sentido, el aprendizaje que se extiende más allá de las técnicas y habilidades profesionales necesita un proceso de formación con un marcado carácter extensivo. El escenario universitario en este caso debería ser un escenario con ruedas para que pueda llegar a toda la comunidad (Marcovitch, 2002).

Los conocidos practicums universitarios no representan exactamente nuestra idea de extensión universitaria como manera de contactar con la comunidad y con la vida profesional que espera una vez obtenido el título. No creemos que sea un escenario oportuno para tratar de enseñar aquello que hemos llamado el aprendizaje de la responsabilidad ni del saberse universitario. El consorcio universidad-comunidad no debería conformarse con un número de horas de prácticas en organizaciones empresariales. Un número de horas irrisorio, teniendo en cuenta el objetivo que se pretende conseguir. La formación de ciudadanos universitarios comprometidos y responsables de sus actuaciones pasa por la sistemática puesta en marcha de auténticas prácticas de formación en contextos comunitarios (McIntyre, 1987). La extensión de la universidad pasa por la virtud de compartir lo que ha sido dado, a saber, el conocimiento y la cultura, y es en este compartir con la comunidad donde ha de tener cabida la formación en la universidad de hoy.

Los estudiantes deberían participar desde el principio en la extensión universitaria. Extensión que se debería concretar en prácticas de formación donde aplicar el conocimiento para que el estudiante deguste y sienta la responsabilidad social que le ha sido otorgada. Al respecto, nos vienen bien las sugerencias que nos llegan desde Bolonia, a saber, ya no va a hacer falta estar sentado en una silla tomando apuntes para ir superando asignaturas. También se puede aprender fuera del aula, en el contexto social y comunitario. Nos advirtió hace siglos el filósofo de Estagira que el aprendizaje para la comunidad debería ser comunitario. 
En definitiva, las fronteras entre la institución universitaria y la comunidad social deberían abrirse más que nunca para que las aportaciones de la primera puedan extenderse a la segunda, y las sugerencias de la segunda se incorporen a la primera. El contacto puntual, empresarial y a veces interesado, debería convertirse en sistemático, social y confiado. De esta manera, la enseñanza universitaria debería organizar un escenario de formación extensivo propio de una universidad abierta y con una función social que cumplir. Quizás es una manera de superar las diferencias abismales que hay entre los fines de la universidad y su paideia (Harvey y Knight, 1996).

\subsection{La reflexión a tientas en la universidad de boy}

El debate, decididamente abierto, sobre formación universitaria parece que será de larga duración. Quizás ha llegado el momento de reconocer que la praxis educativa en Educación Superior ha sido la gran derrotada frente a legislaciones, convenios, investigación, y otros avatares del hecho universitario. Efectivamente, la reflexión sobre el sentido y la forma de ayudar al otro en su proceso de aprendizaje y de desarrollo personal ha brillado por su ausencia en el hecho universitario. Con las excepciones de siempre, la mayoría hemos caído en la cuenta que saber mucho no está relacionado con el arte de saberlo hacer saber a otros. Dos mundos totalmente diferentes han sido encolados a la ligera en el hecho universitario contemporáneo. En otras palabras, el experto en un ámbito de conocimiento con años de experiencia en investigación e innumerables publicaciones ha sido convertido por arte de magia en docente universitario.

No pretendemos poner en duda el valor de la formación docente en investigación, está fuera de toda discusión. Dudamos de la conexión o asociación que sobre lo dicho se establece alegremente con la docencia. Así pues, sabiendo que el aprendizaje ético debería ser objeto de enseñanza en la universidad, surge automáticamente la pregunta: ¿Qué es por lo tanto ser un buen profesor universitario? Las investigaciones sobre la cuestión no sólo no coinciden sino que concluyen que no es fácil encontrar coincidencias (Pozo y Monereo, 2003).

El profesor de la universidad de hoy debería ser el profesional reflexivo que propuso Shön hace unos años (Shön, 1983). Valoramos la actitud reflexiva del docente sobre su misión con los estudiantes, con el conocimiento que se transmite, con la cultura que se construye... a sabiendas que no siempre llegará a conclusiones concretas y cerradas. La actitud reflexiva de quien ha de ayudar e inquietar a otros pasa ineludiblemente por un pensar a tientas. Se trata de alejarse de aquello que se tiene claro y volver a pensarlo tras un tiempo prudencial con nuevas preguntas e ideas renovadas. El profesor que muestra a sus estudiantes la actitud de la duda, los silencios en medio de los discursos con la mirada conscientemente perdida, ofrece lecciones de búsqueda de la verdad y de aceptación de la finitud humana (Melich, 2002). El profesor de la universidad de hoy debería 
perder la mirada y emprender viajes en búsqueda del conocimiento con sus estudiantes, nunca solo, nunca en actitud narcisista. La enseñanza universitaria que parte de la reflexión fomenta el aprendizaje reflexivo y huye de adoctrinamientos y de falsas verdades. La enseñanza universitaria debería ser un ejemplo de razonamiento y de acogida, sobre la base de la compleja realidad, la que hoy vivimos.

\section{PENSAR, Planificar y aCtUAR JUNTOS PARA SUPERAR la CASUALIDAD}

Consideramos que la experiencia universitaria es algo más que un proceso de instrucción y de suma de créditos que ocupa unos pocos años antes de sumergirse en sus quehaceres profesionales. El hecho universitario debería llegar a ser una experiencia de vida, un conjunto de situaciones vividas y sentidas que influyen enormemente en el desarrollo moral y ético de una persona mientras se forma en una profesión. Así pues, el proyecto universitario no es pura y simplemente una condición de posibilidad para ponerse en primera línea de salida a la hora de conseguir trabajo. Esta errónea concepción es la que nos ha llevado a la cultura de la titulitis, del coleccionismo de títulos expedidos por instituciones universitarias, sean cuales sean sus siglas. El proyecto universitario debería ser la formación de ciudadanos profesionales que tomen conciencia de su papel en la comunidad. Las universitas del siglo que ya está aquí necesitan nuevas razones o quizás necesitan recuperar las razones ya olvidadas. Consecuentemente se necesitan nuevas condiciones para las nuevas razones.

Para dar sentido a las nuevas razones, que hemos llamado aprendizaje ético, y concretar las nuevas condiciones, que hemos llamado enseñanza en la universidad proponemos tres acciones que deberían iluminar el hecho universitario ahora que se ve a lo lejos el horizonte Bolonia. Éstas son: pensar, planificar y actuar de manera conjunta.

La comunidad académica junto con la social y profesional, debería plantearse de una vez por todas el finis ultimus de la formación universitaria. Deberíamos pensar juntos. Tarea en la que deberían participar académicos, representantes sociales, culturales y de las administraciones, expertos de las organizaciones empresariales... Un consejo de pensadores donde nadie quede excluido y que trabajen de una manera alejada de manuales, narrativas y monólogos, y se acerque a un ensayo tal y como Montaigne lo pensó (Montaigne, 1986). Creemos que es más importante pensar lo que queda por decir que insistir sobre lo dicho. ¿Qué debería ser la experiencia universitaria para los estudiantes del s. Xx?? ¿Universitariamente hablando, de dónde venimos y hacia dónde vamos? Se trata de plantear preguntas y tratar de encontrar respuestas aunque sea pensando a tientas.

Deberíamos también planificar juntos. Si es verdad que en la universidad nacen los caminos de formación y desarrollo personal no es menos cierto que éstos tienen que seguir más allá de los campus universitarios. No vemos claras las salidas 
puntuales y fugaces llamadas practicum. El hecho universitario y, sobre todo su sentido, se debería extender más que nunca. Los consorcios entre universidades, entidades culturales y sociales, administraciones y organizaciones empresariales se deberían consolidar y planificar de manera conjunta. La filosofía de las prácticas actuales es superable. Las ideas que han sido pensadas en un primer paso deberían ser planificadas en forma de acciones formativas. Las instituciones universitarias ubicadas en sus comunidades respectivas deberian concretar conjuntamente las acciones formativas que representen trabajo personal del estudiante y que desarrollen su capacidad profesional y ciudadana.

Deberíamos por último actuar juntos. Una experiencia como la universitaria no es una acción puntual, ni tan siquiera una suma de éstas. O se le da desde la institución universitaria el sentido de proceso y globalidad para que represente una experiencia de vida o no se lo dará nadie, ni siquiera el propio estudiante. En este sentido el tratamiento psicopedagógico del conjunto de encuentros entre profesor y estudiante resulta esencial. En definitiva, el sentido del conjunto de interacciones que se dan entre profesor y estudiante es el que nos permitirá construir una universidad u otra.

El ciudadano universitario con experiencia universitaria, más que con reminiscencias puntuales y aisladas, ha sido acogido. Ha sido atendido y respetado y se le ha pedido que atienda y respete las normas de la casa, de la universidad. Ha encontrado miradas de atención y actos de ayuda para ir transformándose como persona y como profesional. No será pues casualidad que el estudiante haga lo mismo con otros que se encontrará en el caminar por la vida, quizás en peores condiciones. El sentido de vida que se puede consolidar o hacer nacer en la experiencia universitaria no es cosa fácil de olvidar.

La mirada atenta, la pregunta oportuna, la devolución discursiva del proceso de aprendizaje, la convivencia con personas comprometidas, las buenas maneras en el trato personal, saberse parte de un proyecto comunitario... son las pequeñas acciones que hacen que el aprendizaje ético y la enseñanza universitaria vayan más allá de la casualidad.

\section{BIBLIOGRAFÍA}

Borges, J. L. (1972) Ficciones. Barcelona, Círculo de Lectores.

BRICALL, J. M. (1997) Visión de la Universidad en Europa: problemas diversos, soluciones comunes, en Conferencia pronunciada en el ciclo Los nuevos desafios de la Universidad. Institución libre de Enseñanza. Madrid, ILE, Morata.

CASTELLS, M. (1997) La era de la información. Economía, sociedad y cultura. Vol. I de La sociedad red. Madrid, Alianza.

Claramunt, S. (2002) Orígenes del mundo universitario: de los studia a las universitas, en Busqueta, J. y Pemán, J. (eds.). Les universitats de la corona d'Aragó abir $i$ avui. Barcelona, Editorial Pòrtic, 27-35. 
DerRIDA, J. (2002) La universidad sin condición. Madrid, Mínima Trotta.

EsCOTET, M. A. (2004) Globalización y educación superior: desafíos en una era de incertidumbre, en II Congreso Internacional de Docencia Universitaria. Barcelona, Edicions UB.

EsteBAN, F. (2004) Excelentes profesionales y comprometidos ciudadanos: Un cambio de mirada desde la Universidad. Bilbao, Descleé de Brouwer.

GALBRAITH, J. K. (1982) La era de la incertidumbre. Madrid, Plaza y Janés.

HABERMAS, J. (1954) Teoría de la acción comunicativa. Complementos y estudios previos. Madrid, Cátedra.

- (1984) Ciencia y técnica como ideología. Madrid, Tecnos.

- (1987) Teoria y praxis. Estudios de filosofía social. Madrid, Tecnos.

- (2000) Aclaraciones a la ética del discurso. Madrid, Mínima Trotta.

HARVEY, L. y KNIGHT, P. (1996) Transforming Higher Education. Buckingham, SRHE/Open University Press.

Hoyos, G. (2004) El ethos de la universidad, en EsteBAN, F. (ed.). Universidad, profesorado $y$ ciudadania. OEI, www.oei.org/valoresmono/monografias.

IYANZA, A. (2000) Historia de la Universidad en Europa. València, Universitat de València.

JONAS, H. (1995) El principio de la responsabilidad. Ensayo de una ética para la civilización tecnológica. Barcelona, Herder.

LEvinas, E. (1993) Humanismo del otro hombre. Madrid, Caparrós Editores.

Marcovitch, J. (2002) La universidad (im)posible. Madrid, Cambridge/OEI.

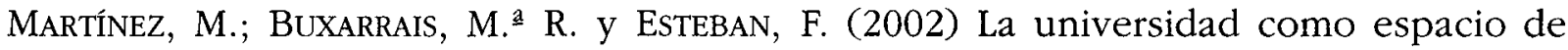
aprendizaje ético, Revista Iberoamericana de Educación, 29, 17-43.

McINTYRE, A. (1987) Tras la virtud. Barcelona, Crítica.

MeLICH, J. C. (2002) Filosofia de la finitud. Barcelona, Herder.

Montaigne, M. (1968) Ensayos completos. Barcelona, Iberia.

Morin, E. (2000) La mente bien ordenada. Barcelona, Seix Barral.

ORTEGA Y GASSET, J. (1930) La misión de la Universidad. Madrid, Alianza.

Pozo, J. I. y MONEREO, C. (eds.) (2003) La universidad ante la nueva cultura educativa. Enseñanza y aprendizaje para la autonomía. Madrid, Síntesis.

PuIG, J. M. ${ }^{a}$ (1996) La construcción de la personalidad moral. Barcelona, Paidós.

QuinTANilla, M. A. (1999) La misión y el gobierno de la universidad abierta, Revista de Occidente, 216, 117-146.

RicoeuR, P. (1900) Sí mismo como otro. Madrid, Siglo XXI.

SaVATER, F. (1996) El contenido de la felicidad. Madrid, Santillana.

SENNET, R. (2000) La corrosión del carácter. Barcelona, Anagrama, 154.

- (2003) El Respeto. Sobre la dignidad del hombre en un mundo de desigualdad. Barcelona, Anagrama.

SHÖN, D. (1983) The reflective practitioner. New York, Basic Books.

TORRALBA, F. (2001) Rostro y sentido de la acción educativa. Barcelona, EDEBÉ.

- (2002) ¿Un altre món és posible? Educar després de l'Onze de Setembre. Barcelona, Edicions 62.

TORRENTS, R. (2002) Noves raons de la Universitat. Vic, Eumo Editorial, 34.

UNESCO (1996) Informe a la UNESCO de la Comisión Internacional sobre la educación para el siglo Xxi presidida por J. Delors, La educación encierra un tesoro. Madrid, Santillana.

- (1997) Hacia una nueva educación superior. CRESALC/UNESCO.

VV.AA. (2002) Joves $i$ valors. Els joves Catalans en l'enquesta europea de valors. Barcelona, Col-lecció d'Estudis de la Generalitat de Catalunya.

ZUBIRI, X. (1986) Sobre el bombre. Madrid, Alianza. 